\title{
Effects of Resistance Training with and without Blood Flow Restriction on Cardiovascular Risk Factors in Overweight Females
}

\author{
Amin Farzaneh Hesari (PhD) \\ Department of Exercise Physiology, \\ Islamic Azad University, Sari Branch, \\ Sari, Iran \\ Akram Ebrahimi (MSc) \\ Department of Exercise Physiology, \\ Islamic Azad University, Sari Branch, \\ Sari, Iran \\ Masoumeh Azizi Zanjani (MSc) \\ Department of Exercise Physiology, \\ Islamic Azad University, Sari Branch, \\ Sari, Iran \\ Solmaz Mahdavi (MSc) \\ Department of Sport Injuries, Faculty \\ of Physical Education and Sport \\ Sciences, University of Arak, Arak, \\ Iran \\ Corresponding author: Amin \\ Farzaneh Hesari \\ Tel: +989113707492 \\ Email: af.hessari@gmail.com \\ Received : 02 Sep 2017 \\ Revised: 01 Jan 2018 \\ Accepted: 13 Jan 2018
}

\begin{abstract}
Background and Objectives: Elevated blood lipid levels are correlated with risk of cardiovascular disease, especially in overweight individuals. The beneficial effect of strength training on lipid profile has been well documented. However, the effect of strength training with flow blood restriction has not been established. The purpose of this study was to compare the effect of eight weeks of strength training with and without blood flow restriction on some of the cardiovascular risk factors in overweight females.

Methods: In this study, 28 overweight females aged 18 to 24 years were randomly assigned into three groups of blood flow restriction strength training (BFRT), traditional strength training (TRT) and control. Participants in the BFRT group performed eight weeks of strength training three sessions per week at $30 \%$ of 1-repetetion maximum, while participants in the TRT group performed the training at $65-00 \%$ of 1-repetetion maximum. Cardiovascular variables, systolic blood pressure and diastolic blood pressure were measured before and after the exercise training. Data was analyzed using descriptive statistics, paired t-test and one-way analysis of variance.

Results: Triglyceride level decreased significantly in the BFRT $(\mathrm{p}=0.022)$ and TRT $(p=0.038)$ groups compared with the control group. Moreover, there were no significant differences in the body mass, waist-to-hip ratio, body mass index, high-density lipoprotein, low-density lipoprotein, and cholesterol levels between the study groups $(\mathrm{P} \geq 0.05)$.

Conclusion: 0ur findings suggest that the BFRT and TRT may not be suitable for preventing or even reversing the physiological changes induced by obesity.

Keywords: Resistance Training, Lipids, lipoprotein, overweight.
\end{abstract}




\section{INTRODUCTION}

Obesity is a major health problem associated with several other risk factors of coronary heart disease such as hypertension, dyslipidemia and glucose intolerance. Body fat distribution is also associated with the risk of cardiovascular disease (CVD). Several factors including abdominal obesity (waist-hip ratio), hypertension, high cholesterol and triglyceride levels, stress and smoking influence the risk of CVD (1). Meanwhile, low blood pressure in normal people significantly reduces the risk of CVD (2).

CVD is the leading cause of death worldwide. According to the statistics of the Iranian Ministry of Health, CVD is responsible for $38.5 \%$ of all deaths in Iran (3). The LDL/HDL ratio can be used for predicting the risk of coronary heart disease, especially atherosclerosis. Furthermore, the cholesterol/LDL ratio is one of the best risk indicators of coronary artery disease (4).

Several studies have investigated the effect of exercise on the risk factors of CVD. Most of these studies indicated that regular exercise has beneficial effects on the risk factors. However, majority of these studies have focused on endurance training and limited number of studies have assessed the effect of strength training. There are also some inconsistencies regarding the effects of strength training on the risk factors of CVD. For instance, a study showed that 10 weeks of strength training significantly reduces the waist-hip ratio (WHR) without affecting LDL and triglyceride levels (5). Meanwhile, another study demonstrated that strength training has beneficial effects by lowering blood pressure and lipid profile (6). Some studied reported that strength training has no effect on the lipid profile $(7,8)$. The inconsistency in the results of these studies could be related to the intensity of training. Strength training with blood flow restriction (occlusion training) has been recently proposed as a new type of strength training. In this type of training, individuals perform strength training at low intensity by fastening a light and flexible rubber band around the upper part of arm or thigh to create a suitable surface pressure (9). Intensity of the trainings is usually equivalent to daily activities and endurable for most people with different physical characteristics. Some studies indicated that training with blood flow restriction has the same advantages of the traditional strength training $(10,11)$.

Most studies conducted on the effect of strength training on the risk factors of CVD have utilized traditional strength training at high intensities, which might not be suitable for all individuals. Strength training with blood flow restriction influences muscle hypertrophy, which can affect the metabolism of fat and carbohydrates (12). In addition, high intensity-strength training might be difficult for some individuals. Therefore, the present study evaluated the effect of eight weeks of strength training with/without blood flow restriction on some risk factors of CVD in overweight females.

\section{MATERIAL AND METHODS}

The study protocol was approved by the Research Ethics Committee of Islamic Azad University of Sari (Iran), and registered at the Iranian Registry of Clinical Trials (IRCT201709436061N1). The study was performed on 28 overweight female volunteers aged 18-24 years with body mass index (BMI) of $>25$. All participants were asked to give written consent, were familiarized with the training program and informed about the possible risks and benefits of the study. They were randomly divided into blood flow restriction training (BFRT, $n=9$ ), traditional resistance training (TRT, $\mathrm{n}=9$ ) and control $(n=10)$ groups. At baseline, BMI, resting heart rate (HR), systolic blood pressure (SBP), diastolic blood pressure (DBP) and lipid profile were evaluated. The tests were repeated for all groups after eight weeks. The participants in the BFR and TRT groups performed regular resistance training for eight weeks, whereas those in the control group did not perform any exercise. All participants were advised to keep their habitual diets, and instructed not to take fried foods and high sugar foods during the intervention period.

The participants performed resistance training three times a week for eight weeks. The training consisted of a 5-minute warm-up, resistance exercise training, and 5-minute cool-down. The warm-up and cool-down were performed by stretching and treadmill walking at a constant speed and inclination. The main workout program comprised of leg extension and biceps, back-leg, triceps and leg press. The movements were focused on utilizing the large muscles of the body. The order of the 
movements was designed in a way to utilize frequently upper and lower body muscles (13). For all movements, 1-repetetion maximum (1RM) was measured for each subject at the beginning of the eight-week training program. The BFR group was subjected to a BFR stimulus and completed 30 repetitions followed by three sets of 15 repetitions $(1 \times 30+3 \times 15)$ at $30 \%$ of 1 RM. The TRT group was not subjected to BFR but performed three sets of 10 repetitions at $65-80 \%$ of $1 \mathrm{RM}$. The participants in the TRT group performed trainings at $65 \%$ of $1 \mathrm{RM}$ for the first two weeks, but the intensity increased gradually by $5 \%$ of $1 \mathrm{RM}$ every two week. The $1 \mathrm{RM}$ was measured every two weeks and then the training intensity was set accordingly. Rest between the sets and the stations lasted 1-1.5 minutes and 3-4 minutes, respectively (13).

In order to determine individual's cuff pressure, in a pilot study, a number of BFR participants with different arm and thigh sizes completed a test to determine the maximum limb occlusion pressure (LOP). In order to establish maximum LOP, the participants were asked to lie down in supine position while a blood pressure cuff was attached to their proximal thigh or arm (14). A Doppler probe (Ultrasound Technologies Ltd, Caldicot, UK) was used to detect the auscilatory pulse at the medial malleolus of the right leg tibia. The blood pressure cuff was inflated to $50 \mathrm{mmHg}$ for 30 seconds and then increased in $40 \mathrm{mmHg}$ increments until the arterial pulse was no longer detectable. The pressure was then decreased until the pulse was detected again. Maximum LOP was determined as the greatest pressure at which the arterial pulse was not detected (15). The BFR protocol placed the participants under $70 \%$ LOP, which has been shown to be a safe and practical training program that enhances strength and hypertrophy $(14,16)$.
Waist and hip circumferences were measured using a measuring tape. BMI was calculated as weight $(\mathrm{Kg}) /$ height squared $\left(\mathrm{m}^{2}\right)$, and SBP and DBP were measured in sitting position using an inflatable cuff and mercury sphygmomanometer before and after the exercise. Blood sample was taken from the antecubital vein following a 12-hour overnight fast. Biochemical tests including total cholesterol (TC), high-density lipoprotein cholesterol (HDL), low-density lipoprotein cholesterol (LDL-C) and triglycerides (TG) were carried out by enzymatic colorimetric method using Pars Azmoon kits (Iran). Lipid profile was determined 24 hours before the training program started (pretest) and 24 hours after the last training session (posttest). It should be noted that all participants were in the same menstrual cycle at the time of sampling.

Statistical analysis was performed in SPSS software (version 20). Differences in the variables were assessed using one-way analysis of variance and Tukey post hoc test. The pretest and posttest data were analyzed using paired sample t-test. The results are expressed as mean \pm standard deviation (SD). P-values less than 0.05 were considered statistically significant.

\section{RESULTS}

Based on the anthropometric characteristics of the participants, there was no significant difference in the body mass $(\mathrm{F}=1.264, \mathrm{p}=0.267)$, BMI $(\mathrm{F}=1.719, \mathrm{p}=0.364)$, WHR ( $\mathrm{F}=0.867, \mathrm{p}=0.543)$, DBP $(\mathrm{F}=1.019$, $\mathrm{p}=0.679) \quad$ and $\mathrm{SBP} \quad(\mathrm{F}=1.179, \quad \mathrm{p}=0.414)$ between the study groups (Table 1). After the eight-week intervention, BMI decreased by $6.63 \%$ and $18.75 \%$ and WHR decreased by $16.63 \%$ and $18.75 \%$ in the BFR and TRT groups, respectively. The variables remained almost constant in the control group.

Table 1- Mean \pm SD of the anthropometric variables in the studv grouns before and after the training program

\begin{tabular}{|c|c|c|c|c|}
\hline Variable & Group & Pretest & Posttest & \\
\hline \multirow{3}{*}{ Body mass (Kg) } & & Control & $68.8 \pm 6.3$ & $68.6 \pm 7.2$ \\
\hline & & BFR & $71.2 \pm 8.2$ & $69.8 \pm 5.8$ \\
\hline & & TRT & $69.9 \pm 6.4$ & $68.7 \pm 8.1$ \\
\hline \multirow{3}{*}{ WHR } & & & & \\
\hline & & Control & $0.9 \pm 0.1$ & $0.9 \pm 0.4$ \\
\hline & & BFR & $1 \pm 0.3$ & $0.92 \pm 0.5$ \\
\hline \multirow[t]{2}{*}{ BMI (Kg/m2) } & & TRT & $0.9 \pm 0.5$ & $0.9 \pm 0.4$ \\
\hline & & Control & $26.7 \pm 2$ & $26.8 \pm 3$ \\
\hline \multirow[t]{2}{*}{ DBP } & & BFR & $27.8 \pm 3$ & $26.9 \pm 4$ \\
\hline & & TRT & $26.4 \pm 4$ & $25.4 \pm 3$ \\
\hline \multirow[t]{6}{*}{ SBP } & & Control & $76.7 \pm 6$ & $77.2 \pm 6$ \\
\hline & & BFR & $77.6 \pm 5$ & $72.1 \pm 7$ \\
\hline & & TRT & $76.3 \pm 5$ & $73.2 \pm 6$ \\
\hline & & Control & $118.7 \pm 9$ & $119.2 \pm 8$ \\
\hline & & BFR & $117.3 \pm 7$ & $108.2 \pm 9$ \\
\hline & & TRT & $118.1 \pm 9$ & $110.6 \pm 8$ \\
\hline
\end{tabular}


No significant difference was observed in the TC $(\mathrm{F}=0.647, \mathrm{p}=0.413), \mathrm{HDL} \quad(\mathrm{F}=1.519$, $\mathrm{p}=0.267)$ and $\mathrm{LDL}(\mathrm{F}=1.914, \mathrm{p}=0.549)$ levels between the three groups (Table 2). Comparison of the results of pretest and posttest showed no significant change in the HDL, TC and LDL levels between the BFR and TRT groups.

However, we found a statistically significant difference in the level of TG $(\mathrm{F}=3.697$, $\mathrm{p}=0.042$ ) between the groups. TG levels decreased significantly in the BFR $(\mathrm{p}=0.022)$ and TRT $(\mathrm{p}=0.038)$ groups compared to the control group.

Comparison of the results of pretest and posttest showed that TG level decreased significantly in the BFR ( $\mathrm{t}=1.129, \mathrm{p}=0.029)$ and TRT ( $\mathrm{t}=2.279, \mathrm{p}=0.034)$ groups.

Table 2- Mean \pm SD of the biochemical variables in the study groups before and after the training program

\begin{tabular}{|c|c|c|c|}
\hline Variable & Group & Posttest & \\
\hline \multirow[t]{3}{*}{ TG (mg/dl) } & Control & $131.4 \pm 21.7$ & $128.7 \pm 24.2$ \\
\hline & BFR & $132.6 \pm 19.1$ & $108.5 \pm 21.3$ \\
\hline & TRT & $134.5 \pm 19.3$ & $105.2 \pm 21.7$ \\
\hline \multicolumn{4}{|l|}{ TC (mg/dl) } \\
\hline & Control & $164.1 \pm 29.2$ & $163.6 \pm 28.3$ \\
\hline & BFR & $171.1 \pm 31.3$ & $159.7 \pm 26.6$ \\
\hline & TRT & $163.8 \pm 28.4$ & $155.2 \pm 25.2$ \\
\hline \multirow[t]{3}{*}{ HDL (mg/dl) } & Control & $46.4 \pm 5.2$ & $46.8 \pm 4.7$ \\
\hline & BFR & $45.2 \pm 6.3$ & $50.8 \pm 7.2$ \\
\hline & TRT & $43.8 \pm 5.2$ & $47.2 \pm 6.1$ \\
\hline \multirow[t]{3}{*}{ LDL (mg/dl) } & Control & $144.7 \pm 19.9$ & $145.1 \pm 17.7$ \\
\hline & BFR & $145.1 \pm 21.3$ & $138.8 \pm 18.5$ \\
\hline & TRT & $139.9 \pm 16.5$ & $132.6 \pm 16.3$ \\
\hline
\end{tabular}

\section{DISCUSSION}

We found that both traditional high intensity resistance training and the low intensity-BFRT significantly decreased TG level compared to the control group. This finding is consistent with reports of some previous studies $(5,6,17)$. However, Heydarpour et al. reported that 10 weeks of resistance exercise had no significant effect on the lipid profile of premenopausal women (18). Hosseini Kakhak et al. also reported that eight weeks of strength training (four sessions a week) at $60-70 \%$ of $1 \mathrm{RM}$ had no significant effect on the lipid profile of obese girls (19). They proposed that longer training periods at higher intensity might have more favorable effects on TG and TC levels. Moreover, Vincent et al. demonstrated that six weeks of strength training had no significant effect on the lipid profile of inactive men (7).

The contradictory results for the level of TG could be due to the association of TG with reduction of body-fat percentage and exerciseinduced alterations in body composition. Similarly, the response to training programs depends on several factors including age, gender, basic TG levels, physical fitness and duration, intensity, volume and diversity of the training program $(21,22)$. Energy expenditure has been considered as another factor that could significantly reduce TG level following trainings. It seems that daily energy expenditure is the primary determinant of $\mathrm{TG}$ level (23). Therefore, resistance training with blood flow restriction might increase the rate of basic metabolism caused by increased muscle mass.

Previous studies showed that resistance training could reduce TG level by increasing activity of lipoprotein lipase. The enzyme releases fatty acids from the TG in fat and muscle tissue, which in turn increases the catabolism of TG and TG-rich lipoproteins and facilitates the removal of TG from the bloodstream. What justifies this mechanism is that such decrease in the TG level takes place even with no change in the body composition (24).

It seems that angiogenesis is the main mechanism for the long-term effects of BFRT (25). Angiogenesis coordinates functions of endothelial cells toward formation of new blood vessels from pre-existing vessels. This physiological adaptation mechanism compensates for increased metabolic demand of the tissue by increasing TG uptake from the blood. Release of metabolites such as 
adenosine, nitric oxide and bradykinin due to blood flow restriction could be another possible mechanism. Hypoxia and ischemia caused by occlusion training could increase vascular endothelial growth factor (VEGF) and endothelial progenitor cells, which might improve the endothelial function (26). In addition, tissue ischemia and shear force are both major stimulators for the release of endothelial progenitor cells from the bone marrow into the blood. Moreover, increase in hypoxia-1 induced factor (HIF-1) is known to regulate the expression of $V E G F$ gene. Studies indicate that HIF-1, even in the absence of hypoxia, could be activated by factors such as nitric oxide (27). Furthermore, it has been found that mechanical pressure due to muscles movement stimulates nitric oxide release and subsequently plays a key role in activation of VEGF signaling pathway (27). It can be concluded that the increased capillary bed and blood flow to the muscles and other tissues due to angiogenesis might contribute to the reduction of TG level.

In this study, we observed that the strength trainings with blood flow restriction had no significant effect on body weight and BMI. The weight remained constant possibly due to increased muscle mass and decreased fat mass. However, since body fat was not evaluated in

\section{REFERENCES}

1. Kelly T, Yang W, Chen C, Reynolds K, He J. Global burden of obesity in 2005 and projections to 2030. Int J Obes. 2008; 32(9): 1431-7. doi: 10.1038/ijo.2008.102.

2. Parente B, Guazzelli I, Ribeiro MM, Silva AG. Obese children lipid profile: Effects of hypocaloric diet and aerobic physical exercise. J Endocrino Metabolism. 2006; 50(3): 499. 504

3. Khadem vatan K, Haghparast F, Eftekhar E, Noroz zade J. Pro oxidant and antioxidant status in patients with coronary artery disease proven angiographically. Hormozgan. Medical Journal. 2009; 12(4): 2316.[Persian]

4. Wang Z, Nakayama T. Inflammation, a link between obesity and cardiovascular disease. Mediators Inflamm. 2010; 53: 59-18. doi: 10.1155/2010/535918.

5. Correa C, Teixerira B, Bittencourt A, Lemos L, Radalli R, Pinto R. effect of high and low strength training on muscle strength and lipid profile in postmenopausal women. J of Exercise Science \& Fitness. 2014; 12(2): 62-67.

6. Gomez J, Rodríguez G, Royo A, Martínez-Redondo R, Luis A, Casajús J. Effect of endurance and resistance training on regional fat mass and lipid profile. Nutr Hosp j. 2013; 28(2): 340-346. doi: 10.3305/nh.2013.28.2.6200. our study, verification of this statement requires more detailed evaluations. Since the TG reduction was observed only in the BFR and TRT groups, it is difficult to determine whether this change was induced by exercise or normal day-to-day variation. Although we instructed the participants to maintain their normal diet during the training period and prior to blood collection, we did not monitor or restrict their diet, which could be considered a limitation for the present study.

\section{CONCLUSION}

Our results indicate that eight weeks of low intensity-BFRT and high intensity-TRT reduce TG level but do not improve TC, HDL and LDL levels in overweight females. For this reason, BFRT and TRT may not be suitable non-pharmacological interventions for preventing or reversing the physiological changes induced by obesity.

\section{ACKNOWLEDGMENTS}

The authors would like to thank all colleagues in the Islamic Azad University of Sari and all participants in the study for their assistance and cooperation.

\section{CONFLICT OF INTEREST}

The authors declare no conflict of interest.

7. Vincent KR, Braith RW, Bottiglieri T, Vincent HK, Lowenthal DT. Homocysteine and lipoprotein levels following resistance training in older adults. Prev Cardiol j. 2003, 6(4): 197-203.

8. Atashak A, Jafari A, Azarbaijani M. The Influences of long-term resistance training on Adiponectin and lipid profiles levels in obese men. Razi Journal of Medical Sciences.2011, 18 (86): 23-31. [Persian]

9. Kawada SH, Ishii N. Skeletal muscle hypertrophy after chronic restriction of venous blood flow in rats. Med Sci Sports Exerc. 2005; 37(7): 1144-1150.

10. Madaram H, Sasaki K, Ishi N. Endocrine responses to upper and lower-limb resistance exercises with blood flow restriction. Acta Physiol Hung. 2010; 97(2): 192200. doi: 10.1556/APhysiol.97.2010.2.5.

11. Laurentino G, Ugrinowitsch C, Aihara A Y, Fernandes A R, Parcell A C, Ricard M, et al. Effects of strength training and vascular occlusion. Int $\mathrm{J}$ Sports Med: 2000, 29(8): 664-7. doi: 10.1055/s-2007-989405.

12. Suga T, Okita K, Morita N, Yokoto T, Hirabayashi $\mathrm{K}$. Intramuscular metabolism during low-intensity resistance exercise with blood flow restriction. J Appl physiol. 2009, 106(4): 1119-24. doi: 10.1152/japplphysiol.90368.2008. 
13. Hosseini Khakhk SA, Sharifi A, Hamedi Nia MR. A comparison of the effect of traditional resistance training with resistance training with vascular occlusion on muscular function and cardiovascular endurance in young females. Sport Bioscience J. 2011; 3(10): 95-114. [Persian]

14. Scott BR, Loenneke JP, Slattery KM, Dascombe BJ. Exercise with blood flow restriction: An updated evidence-based approach for enhanced muscular development. Sports Medicine. 2015; 45(3): 313-25. doi: 10.1007/s40279-014-0288-1.

15. Lixandrão $M$ E, Ugrinowitsch $C$, Laurentino $G$, Libardi C A, Aihara A Y, Cardoso F N, et al. Effects of exercise intensity and occlusion pressure after 12 weeks of resistance training with blood-flow restriction. European Journal of Applied Physiology. 2015; 115(12): 2471-80. doi: 10.1007/s00421-015-3253-2.

16. Mattar MA, Gualano B, Perandini LA, Shinjo SK, Lima FR, Sá-Pinto AL, et al. Safety and possible effects of low-intensity resistance training associated with partial blood flow restriction in polymyositis and dermatomyositis. Arthritis Research \& Therapy. 2015, 16(4): 473-451.

17. Mogharnasi M, Bagheri M. The Effect of 12 Weeks of Circuit Resistance Training on C-Reactive Protein and Lipid Profile in Inactive Women. Sport Biosciences J. 2014, 6(2): 233-44. [Persian]

18. Heydarpour P, Fayazi S, Haghighi S. Resistance Training Effect on Lipid Profile and Body Fat Percentage of Premenopausal Women. Jundishapur J Chronic Dis Care. 2015; 4(2): 25-34.

19. Hosseini Kakhk SA, Amiri Parsa T, Azarnive M, Hamedinia MR. The Effect of Resistance Training, Aerobic Training and Detraining on the Lipid Profile and CRP in Obese girls. Journal of Sabzevar University of Medical Sciences. 2011, 18(3): 187-197. [Persian]
20. Banz WJ, Maher MA, Thompson WG, Bassett DR, Moore W, Ashraf M. Effect of resistance versus aerobic training on coronary artery disease risk factors. Experimental Biology and Medicine. 2003; 228(4): 434440 .

21. Thompson PD, Tsongalis GJ, Seip RL, Bilbie C, Miles M, Zoeller R, et al. Apolipoprotein E genotype and changes in serum lipids and maximal oxygen uptake with exercise training. Metabolism J. 2004; 53 (2): 193-202.

22. Eliakim A, Makowski GS, Brasel JA, Cooper DM. Adiposity, Lipid levels and brief endurance training in nonobese adolescent male. J sports Med. 2000; 21(5): 332. 337.

23. Davis G, Bartoli W, Durstin L. Effects of acute exercise intensity on Plasma Lipid and apolipoprote in intrained runners. J of Appl Physiol. 1992; 72(3): 91419.

24. Linda MH, Serge P, Duvillord V. Lipid and lipoprotein profile, cordiovascular fitness, bodycomposition and deit during and after resistance, aerobic and combination training in young. Women Eur J Appl Physiol. 2000; 82(5-6): 451-458.

25. Jones H, Hopkins N, Bailey B, Thijssen H. Seven-day remote ischemic preconditioning improves local and systemic endothelial function and microcirculation in healthy. American Journal of Hypertension. 2014; 27(7): 918-25. doi: 10.1093/ajh/hpu004.

26. Jones H, Green D J, George K, Atkinson G. Intermittent exercise abolishes the diurnal variation in endothelial-dependent flow-mediated dilation in humans. Am J Physiol Regul Integr Comp Physiol. 2010; 298(8): 427-432. doi: 10.1152/ajpregu.00442.2009.

27. Semenza GL, Agani F, Booth G, Forsythe J, Iyer N, Jiang BH. Structural and functional analysis of hypoxiainducible factor 1. Kidney Int. 1997, 51(2): 553-5. 